\title{
Contributory factors that lead to increase of mother-to-child transmission of HIV in Capricorn district of Limpopo Province, South Africa
}

\author{
Dorah U. Ramathuba, Maria R. Ramashala, Ntsieni S. Mashau \\ University of Venda, South Africa
}

\begin{abstract}
Introduction: Mother-to-child transmission (MTCT) of human immunodeficiency virus (HIV) remains the most prevalent source of pediatric HIV infections. The prevalence of HIV is high among South African women of reproductive age, and the transmission of HIV from mothers to children is a serious concern. The study explored contributory factors, which lead to an increase in mother-to-child transmission of HIV at selected clinics in Capricorn district of Limpopo Province.

Material and methods: The study adopted a qualitative descriptive exploratory design to uncover factors leading to MTCT of HIV. The target population were professional nurses trained in primary healthcare. Purposive sampling technique was used to sample eighteen participants from Seshego Zone clinics in Capricorn district. Unstructured interviews were conducted and audio-recorded. The transcribed data was analyzed using Tesch's' method of data analysis, and presented in themes and sub-themes. Ethical considerations were ensured throughout the study as well as trustworthiness of findings.

Results: The study revealed that poor socio-economic status, traditional and religious beliefs, and lack of knowledge of patients were the major factors contributing to an increase of MTCT of HIV as well as system-related factors. However, the study indicated high knowledge about MTCT and HIV among the nurses.

Conclusions: The study revealed that patient-related factors cause high prevalence of MTCT of HIV. For community health awareness, it is highly recommended to encourage women to abandon their beliefs in order to promote PMTCT.
\end{abstract}

HIV AIDS Rev 2021; 20, $1: 39-45$ DOI: https://doi.org/10.5114/hivar.2021.105111

Key words: child, factors, HIV/AIDS, mother, transmission, professional nurses.

\section{Introduction}

Human immunodeficiency virus (HIV) continues to be a major global public health issue. In 2015, an estimated 36.7 million people were living with HIV (including 1.8 mil-

Address for correspondence: Dorah U. Ramathuba, University of Venda, South Africa, e-mail: dorah.ramathuba@univen.ac.za

lion children), and a global HIV prevalence was $0.8 \%$ [9]. The vast majority of this number live in low- and middle-income countries. Since the start of the epidemic, an estimated 78 million people have become infected with HIV, 35 million people have died due to AIDS-related illnesses [32], and

Article history:

Received: 07.11.2019

Received in revised form: 19.11.2020

Accepted: 19.11.2020

Available online: 30.03 .2021
International Journal of HIV-Related Problems

HIV \& AIDS

R e vi e w 
an estimated 25.5 million people living with HIV are inhabiting sub-Saharan Africa. Mother-to-child transmission of HIV (MTCT) accounts for about $90 \%$ of HIV infection in infants and young children. It has been reported that over 370,000 infants acquire HIV infections globally each year, with an approximately over 1,000 children acquiring HIV every day [30]. This data represents a decline of $45 \%$ and $71 \%$, respectively, since 2000 .

Globally, in 2015, there were 0.3 new HIV infections per 1,000 uninfected people, and among children under 15 years of age, there were 0.08 new HIV infections. The recent fact sheet by UNAIDS [30] reported that as a result of intensified HIV prevention services, there was a $70 \%$ decline in the number of new HIV infections among children between 2000 and 2015. Despite this significant progress, the number of children becoming newly infected with HIV remains unacceptably high. About 150,000 (110,000-190,000) children became infected with HIV in 2015, down from 490,000 $(430,000-560,000)$ in 2000 [30]. This data represents a decline of $45 \%$ and $71 \%$, respectively, since 2000 .

The risk of a mother living with HIV passing the virus to her child can be reduced to $5 \%$ or less if she has access to effective antiretroviral therapy during pregnancy, delivery, and breastfeeding.

It has been reported that over 370,000 infants acquire HIV infections globally each year with an approximately over 1,000 children acquiring HIV every day [30]. HIV infections in infants and young children occur during pregnancy, labor, and delivery, and postnatally through breast feeding. In breastfeeding populations, between 15 and 45\% of infants born to HIV-infected mothers acquire HIV infection without any intervention [33].

Prevention of mother-to-child transmission (PMTCT) of HIV is an intervention, which provides mothers with counseling, antiretroviral (ARV) drugs, and psychological support to help preventing the infants from acquiring HIV infection. The intervention is aimed at eliminating pediatric HIV to ensure no baby is born with HIV infection by 2015 [31]. PMTCT services ensures primary prevention of HIV among women of reproductive age, appropriate counseling of HIV-infected women to enable decision about their future reproduction to prevent unplanned pregnancies, ensure pregnant women receive HIV testing and access to ARV drugs for their health and prevention of infection to babies [31].

The sub-Saharan Africa (SSA) is highly affected by HIV, with an estimated 22.5 million people living with HIV/ AIDS, of which 12 million are women and girls [30]. A related study also revealed that a number of infections and people receiving antiretroviral drugs have increased, and that $90 \%$ of HIV infections, which occurred in sub-Saharan countries were as a result of mother-to-child transmission (MTCT) during pregnancy, labor, delivery, or breastfeeding [8]. Bhardwaj et al. [4] in their study showed that transmission during pregnancy was 5-10\%, during labor and delivery was $10-20 \%$, and during breastfeeding 10-15\%. Without interventions, there was a $20-45 \%$ chance that a baby born to an HIV-infected mother would become infected.
Statistics has indicated that Republic of South Africa (RSA) has the highest number of HIV/AIDS infections worldwide, with the total number of people living with the virus estimated as approximately 5.51 million in 2014 [28]. The disturbing fact is that HIV prevalence amongst antenatal clients across the country is also increasing. South Africa is one of the most burdened countries in the world, where child and infant mortality rates have risen, mainly due to the load of pediatric HIV disease, with more than half of children' deaths resulting from HIV/AIDS [15].

However, despite this general decline, particularly in South Africa's Seshego clinics, the statistical presentation of poor antenatal care (ANC) visits by pregnant women can be associated with high-rate of new HIV infections resulting from mother-to-child transmission. The district health information system (DHIS Capricorn, Limpopo, 2016) reported that ANC firstly tested HIV-positive rate between 2015 and 2016 was $16.1 \%$, and those already on ART indicated $24.6 \%$. The statistics shows that the positivity rate for ANC is high as compared to previous years.

\section{Material and methods}

This qualitative study was conducted using a range of methods with qualifying words and descriptions, to record and investigate various aspects of social reality [6]. The study design explores the perspectives of professional nurses regarding prevention of mother-to-child transmission of HIV in their naturalistic setting as well as helps to understand the reality from the perspective of study participants.

The present study was of qualitative descriptive exploratory design and focused on contributing factors to motherto-child transmission of HIV. Exploratory was used because the researchers wanted to further investigate what exactly contribute to the increase of infection in infants. The study was conducted at selected clinics of Capricorn District of Limpopo Province in South Africa. Capricorn district clinics was purposively chosen because of observed problems in mothers not adhering to treatment regimens. The target population were professional nurses who are specializing in diagnosing, dispensing medication for patients, and monitoring adherence to medication of mothers of HIV-infected children. Non-probability sampling focused on sampling techniques where investigated units were based on the judgment of a researcher in 4 clinics around Seshego Zone, with professional nurses purposively sampled. There were thirty-two professional nurses implementing PMTC of HIV, and majority of them had agreed to participate in the study. A purposive sampling technique was used to interview participants, and data saturation was reached with the eighteenth participant. Semi-structured interview is a method of vetting prospective employees in person, taking a flexible approach and permits questions to arise in response to the dialogue [22]. A semi-structured interview was developed based on the study's specific objectives to guide the researchers during the interview process. A central ques- 
tion was posed in the beginning of the interview process: "What are the factors contributing to the increase of MTCT in relation to patient care and healthcare system?" Probing techniques were used to allow participants to freely explain their experiences deeply. The interviews were conducted individually in English language since the nurses were fluent in English. Face-to-face individual interviews were conducted, and lasted for maximum forty minutes. The interviews were audio-taped, with field notes taken. Data saturation was reached with eighteen female professional nurses attending pregnant mothers. Data collection started in April 2018 and lasted till July 2018.

\section{Data analysis}

Data analysis for qualitative studies progresses through classification of ideas, themes, topics, activities, and types of people as well as other categories relevant to the study were recorded [26]. Audio-taped interviews were transcribed into verbatim. Data was analyzed in a qualitative manner using Tesch's eight steps to identify themes and sub-themes. Recorded interviews and the transcripts were utilized to organize raw data, and concepts with similar attributes were identified and grouped together. Furthermore, common themes were identified from the actual words of participants. The themes abbreviated as codes were then written next to the appropriate segments of transcript. The themes and subthemes were developed and verified by the team members.

\section{Trustworthiness}

The aim of trustworthiness in a qualitative inquiry is to support the argument that the inquiry's findings are "worth paying attention to". To ensure the trustworthiness and rigor of qualitative findings, qualitative researchers considered dependability, credibility, transferability, and confirmability as trustworthiness criteria. According to Anney [1], qualitative research data collection requires the researcher's self to immerse in participants' world, which was achieved through prolonged interviews lasting approximately forty minutes, then, going back to the participants for data verification and attending the research site within four months resulted in building a relationship. Triangulation helps the investigator to reduce bias, and cross examines the integrity of participants' responses. For the purpose of this study, data was collected using different research instruments, including note taking, audio taping, and participants observation. Dependability of findings was established using a stepwise replication and code re-code approach. Stepwise replication is a qualitative research data evaluation procedure, where two or more researchers analyze the same data separately and compare the results [2]. Conformability of qualitative inquiry was achieved through an audit trial and reflexive journal, in which transcripts, audio-recordings, and field notes were made available to supervisors to confirm the findings.
In order to ensure transferability of qualitative inquiry, the researcher must collect 'thick' descriptive data that allows comparison of this context to other possible circumstances, to which transfer might be considered, and produce a profuse description of the context to make a judgment appropriate to other possible contexts. Dense description of the research process and findings was used to guarantee the transferability of study findings.

\section{Ethical considerations}

The University Higher Degrees Committee (UHDC) at the University of Venda recommended the study, which was approved by the University Research Ethics Committee (No. SHS/18/PH/03/1204). The ethical clearance was then presented to the Department of Health of Limpopo Province and Seshego Zone clinics' management to permit the access of participants. The researcher ensured that all the essential information, such as the purpose and significance of the study as well as voluntary participation was included in a consent form, which was sighed before the study. Moreover, the participants were informed they could withdraw at any time during the study if they do not wish to participate in it any longer. The participants were also informed that the information collected for the research project would be kept confidential, and their sensitive and personal data was protected by pseudo codes.

\section{Presentation of findings}

Study findings were presented in themes and sub-themes as identified during analysis, and the verbatim supporting the findings would be presented also.

\section{Demographic profile of participants}

Eighteen female professional nurses were interviewed, with eight $(n=8)$ having a diploma in nursing science, five holding a degree in nursing, three with honors in nursing, and two participants attained master's in nursing. The distribution of study participants from four clinics in Seshego Zone revealed that the participants were well-experienced, with majority of ten years and above experience and with a small number $(n=4)$ of less than ten years professional nursing experience.

Theme 1: Health system-related factors contributing to MTCT of HIV.

A number of healthcare system contributory factors were identified, including lack of proper facility, poor service delivery, and inaccessibility.

\section{- Lack of proper and well-equipped facilities}

The study identified the lack of proper facilities as a contributor to the increase of MTCT. The participants pointed that programs like PMTCT needs a conducive environment, 
Table 1. Presentation of findings

\begin{tabular}{l|l}
\hline Themes & Sub-themes \\
\hline $\begin{array}{l}\text { Health system-related factors contributing to MTCT } \\
\text { of HIV }\end{array}$ & $\begin{array}{l}\text { Lack of proper and well-equipped facility } \\
\text { Poor service delivery contributes to the increase of MTCT of HIV } \\
\text { Long distance to travel }\end{array}$ \\
\hline $\begin{array}{l}\text { Patient-related factors contributing to the increase } \\
\text { of MTCT of HIV }\end{array}$ & $\begin{array}{l}\text { Traditional and religious beliefs and subjective norms } \\
\text { Socio-economic status of mother increases the risk of MTCT of HIV } \\
\text { Fear of stigma increases MTCT of HIV }\end{array}$ \\
\hline
\end{tabular}

Source: Ramashala, MR, 2019, Factors contributing to mother-to-child transmission (MTCT) of HIV in Seshego Zone clinics, Capricorn district in South Africa, $M P H$, University of Venda, Limpopo

in which the right of an individual for privacy and confidentiality is ensured.

"According to myself, I think we need enough infrastructure, the infrastructure we are working in are not suitable for offering this PMTCT because there is no privacy; we are working in an open space, the consulting rooms are few as they are only four, so there is no privacy, it is not easy to ask questions in front of those who are not infected" (Participant No. 13).

"The rooms are small and not enough, so I think if they can be upgraded, its better to cater for such a sensitive program because sometimes one room caters for two nurses with different patients" (Participant No. 10).

- Poor service delivery contributed to an increase in MTCT of HIV. Shortage of trained professional nurses to provide PMTC

Efficiency of service is rated by turnaround time to provide the required services; due to shortage of trained professional nurses, there are delays in providing patient care. Clients are waiting for a long time resulting in poor adherence to prescribed treatment regimens and some leaving the clinic without getting help because of long ques.

"The waiting time normally is within 3 hours and I think it is too long for our patients, especially pregnant women because they complain that they are going to work or do other activities, such as chores, but now they have to stay long" (Participants No. 12 and 17).

"Shortage of staff is another reason, because the waiting time even gets worse; some mothers are reluctant to come to the facility as they will wait for long hours, since one women servicing takes approximately an hour" (Participant No. 2).

- Long distance to travel

The study revealed that the distance to travel was very long, which varied from approximately five to twenty kilometers and poor transportation, as some patients needed to cross rivers in order to get to the facility.

"5-20 km, people need other people to accompany them to the clinic, if they fail maybe they don't come, and others are crossing the river. During rainy seasons, they don't come to the clinic" (Participant No. 9).

"The radius for people that we are supposed to serve is $5 \mathrm{~km}$, but people coming from far (about $20 \mathrm{~km}$ ) is a problem, as some of them come at 4 am and wait in line, and when we come at 7 a.m., the person is having ticket number 120" (Participant No. 7).

Theme 2: Patient-related factors that contribute to the increase of MTCT of HIV.

Patient-related factors that contribute to MTCT of HIV were identified as complex and varied from area to area and from family to family.

- Traditional and religious beliefs, and subjective norms

The study observed that the majority of mothers who came to the clinic, come for primary healthcare after they have exhausted their traditional healers and faith healers' remedies.

The following opinion were obtained from participant No. 6: "Yes, culturally, our patients think that, let me give an example, when the woman is pregnant, the patient will take time to come to the clinic, she will start ANC at 30 weeks, we take blood and find that the patient is HIV-positive; you will think that if the patient have come earlier, we could have protected the baby, so culturally, our patients believe in traditional healers and prophets. Our patients want to use traditional medicine, they think traditional medicines are the best, they go to traditional healers and use their medicines; they think those are better than the western medicines, which they don't take".

Another participant indicated patriarchal issues: "because the man is still the head of the family; if men refuses condoms, even if they know they are HIV-positive, there is nothing they can do. Even when the mother is breastfeeding, you find that the mother is on ARVs and the husband is not on ARVs, but still he wants to sleep with the woman without condom...so you see the risk of the baby is high. The other thing is traditional use of medicine, that is how the culture affects us. They are using ARVs and herbal medicines they end up with high VL" (Participants No. 4 and 5).

- Socio-economic status of mother increases the risk of MTCT of HIV

The participants indicated that most of the mothers are from poor families with socially ill backgrounds, where some does not have a steady income, as they only relay on a social grant.

"It does affect the program; you give them the dates and they don't have money to come or the husband did not get paid, that is why they did not come in previous month; so 
maybe you were supposed to do something to the baby or give a medication to the mother" (Participant No. 11).

"They have many children because they depend on child grant and they don't eat well-balanced food because they cannot afford it; they are unable as they are not working, and unborn baby become malnourished, resulting as a pre-term delivery" (Participant No. 3).

\section{- Fear of stigma increases MTCT of HIV}

The study revealed stigma as a key to high-rate of MTCT of HIV in rural communities, and is worse when the mother is on treatment and they feel stigmatized to take treatment on time or in the presence of family members.

"They also don't disclose their status as they have the feeling of being discriminated and if they don't disclose, they won't take treatment on time because if there are family members in the sitting room and is time for medication, it will be difficult because they don't want to be seen" (Participants No. 15 and 13).

"As we all know, taking ARVs is not easy, they need love and support because there are side effects and stigma involved, but if the person receive support, it makes things easier. Their viral load are high because they do not take treatment consistently and openly because of fear of discrimination, and the VL is high, which affect the unborn" (Participant No. 9).

\section{Discussion}

The study findings revealed the lack of proper facility, shortage of trained health professionals, and inaccessibility as the main health system factors. The healthcare system in South Africa is being mirrored by problems of unequal distribution of resources due to urban-rural disparity; there are shortages of trained and skilled health professionals, which makes the services to be inefficient. Majority of rural hospital and clinics are in deteriorating state, as they were built during the apartheid era and have not been improved ever since. The infrastructure compromises provision of care as there is no privacy and confidentiality while delivering the services. PMTCT needs a conducive environment, where the right of individuals to privacy and confidentiality is ensured. In a similar study in Pretoria, Tshwane by Ogbonna et al. [20] revealed that availability of adequate facility and equipment for PMTCT program is essential to ensure good quality and sustainability of PMTCT services provided to the clients. Long waiting period characterized by long queues was revealed to be among the health system factors that contribute to an increase of MTCT of HIV. The majority of black population live far away from towns and metropole areas, in rural Capricorn district, where most of the people are poor and uninsured, resulting in having to wake up in early hours of dawn and walk to primary healthcare facilities. Nurses are in the forefront of public health services providing care to majority of the population who are poor and living in remote rural areas. Working conditions of rural areas force nurses to resign and leave to work in urban metropoles, abandoning the already reduced staff who is unable to cope with the workload of patients waiting for prolonged time to be serviced. This is in line with Balira et al. [3] who indicated that long waiting period was caused by the shortage of workforces, such as a limited number of counsellors, midwives, and laboratory personnel, affecting the implementation of PMTCT program. Simba et al. [24] also indicated that inadequate and inaccessible voluntary and counseling centers, coupled with long distance to the centers and long waiting lines, acted as barriers to PMTCT service uptake. Furthermore, in a study conducted in Ethiopia, Merga et al. [13] concluded that the scaling of PMTCT services needs adequate number of trained workforce, as staff shortage influences the PMTCT services quality and de-motivates pregnant women to uptake PMTCT services.

The South African health system is structured as national, provincial, and districts', and resource allocation is also based on the type of facility. Most rural hospitals are district hospitals, which are not adequately financed and together with their primary healthcare services, they are inefficient, ineffective, and unequitable healthcare providers.

The study findings revealed that the distance to travel was very long and varied from approximately five to twenty kilometers, and poor transportation was noted as some patients were from remote areas. With the restructuring of South African health services after 1994, the government indicated that primary healthcare services should be accessible within five kilometers, with services available to provide clients with a variety of facilities by different healthcare professionals. However, this is far from reality, as the public sector is serving a far more geographically dispersed population. The results of the present study are in line with findings of Lankowski et al. [12] where they found that in sub-Saharan areas, PMTCT services are inaccessible to most of pregnant women in need, and particularly with no referral system in place. A study by Clouse et al. [5] conducted in South Africa provided more information on this issue, as it further indicated that HIV pregnant women living near health facility are more likely to be utilizing PMTCT services than those residing far from facility.

Regarding patient-related factors, the findings established that the risk of MTCT of HIV was increased because of pregnant mother's cultural and religious beliefs. It was revealed that pregnant women trust traditional healers and faith healers, and consult them first before seeking professional healthcare. Cultural perceptions and experiences about disease and illness have implications on individual's pattern perceived, and engagement in health-related behaviors and health-seeking behaviors. In Capricorn district, most segments of the population still hold a worldview that illness and health are strongly influenced by spiritual and religious factors that may ultimately affect health outcomes. Ethno-medical approaches, such as the use of herbs, holy oils, and water, are prevalent among the population of Capricorn and influences health outcomes negatively, as women neglect treatment regimen resulting in treatment failure. Mendoza and Lopez [14] demonstrated that many seriously ill patients turn to their spiritual beliefs to cope with their ill- 
nesses and make important medical decisions, and that most patients desire integration of spirituality in their medical care. Emphasis is one of the physical, psychosocial, and spiritual facets of humanity, as an approach to heal the patient.

These findings were in the contrary with a situation in Europe and America, where it was found that cultural beliefs did not prevent black Africans living in London from accessing HIV testing or starting antiretroviral therapy, according to a study by Flint [7]. The author further stated that strong religious beliefs about faith and healing did not act as a barrier to access HIV services or antiretroviral treatment.

Most of the mothers in the study were from poor families and socially ill backgrounds, where some did not have steady income and relied only on social grant. Rural communities are burdened by poverty due to limited employment opportunities and most households are female-headed. Additionally, HIV/AIDS further perpetuates poverty because of loss of employment to those infected or affected members of the family member, resulting in loss of household income. In a study, Schnake and Sommers [25] indicated that pregnant women who lived in poverty faced many barriers to access prenatal care, structural barriers (i.e., lack of transportation, no telephone), healthcare provider shortages, cultural and linguistic barriers, and high levels of stress. Furthermore, the authors observed that poverty leads uncontrolled maternal health conditions, such as diabetes, hypertension, anemia, and obesity, as these conditions can lead to poor PMTCT among pregnant mothers.

The present study further revealed stigma as the key to the high-rate of MTCT of HIV in rural communities served by the selected clinics, where societal acceptance is still poor. People who are living with HIV/AIDS tend to internalize stigma, which subsequently results in negative individual level health consequences, ranging from depression, isolation, to inadequate medication and follow-up visits adherence. Community members also stigmatize a person living with HIV/AIDS through interpersonal relationships processes that are defined by social structure and social relationships, which occur in a community, and these interpersonal processes affect the health outcomes of mothers. Katz et al. [11] revealed that the main barrier was not only fear of knowing one's HIV status, but also fear of being stigmatized. Stigma at an individual level may be a barrier to access and utilize PMTCT services by pregnant women in Africa. A cross-sectional study in Tanzania conducted among pregnant women in 2010 pointed out that the fear of HIV-related stigma was one of the causes for refusal of HIV testing provided by ANC services [16].

The current PMTCT guidelines recommend that all pregnant women should be tested at the initial ANC visit. For those tested negative, a retest at 32 weeks gestation would be taken, and information should be provided on how they can remain negative by practicing safe sex at all times [27]. The findings revealed that women did not disclose their status or that of their babies due to fear of anticipated stigmatization. It is vital that social support be provided to mothers to promote trusting relationship. When women anticipate stigma, they may self-isolate and miss the opportunity for a support, such as visiting healthcare services and receiving healthcare and family supports. Supporting women would increase adherence and improve PMTC resulting in reduced infant morbidities. Family support, which is important in PMTCT programs, also provides optimal access to preventive strategies, such as adherence to ARV therapy. For example, in Lagos, disclosure of HIV status and subsequent treatment support from partners was reported to influence the level of adherence to ARVs among pregnant women [10].

\section{Recommendations}

The following recommendations and conclusions of the present study:

- The Department of Health should improve the infrastructure at primary healthcare level, especially consultation rooms to respect the rights of patients for privacy and confidentiality.

- Training of more nurses on PMTC should be accelerated in order to reduce the acute shortage of implementation of the program.

- Efforts should be made to adapt audience-specific informational and educational materials, strengthen existing community involvement, and address patient-related factors, including unknown maternal HIV infection status before pregnancy, late commencement of antenatal care services, unplanned pregnancies, and low levels of educational status.

- There is also a need to increase family-oriented and culture-friendly community-based PMTCT programs and improve performance to expedite projects and optimize limited resources; it could be used to tackle key challenges encountered during delivery of PMTCT services.

- Women living with HIV should be encouraged to disclose their HIV status to their partners, and should be assisted to overcome barriers, such as retaining women in care, which is responsible for poor treatment adherence through dedicated efforts.

- To increase their knowledge and awareness about PMTCT, information about the program should be given to all women, especially to women in reproductive age. This information could be provided through couple counselling or campaigns to sensitize all women to the issue.

\section{Conclusions}

Findings of the present study revealed that there are still problems regarding effective implementation of PMTC related to system factors, such as long queues, long waiting hours and distances, and poor roads as well as personal factors, such as self-stigmatization, family and community stigmatization, leading to poor service delivery. Factors that nurture an individual, including family contexts are crucial to the success of PMTCT service uptake, such as HIV testing, infant feeding choices, and adherence to ARV's. 


\section{Acknowledgements}

The authors would like to convey their gratitude to the professional nurses participating in the study, the Department of Health, Limpopo Province, Capricorn District, Primary Healthcare for their support.

The project was funded by University research fund.

\section{Conflict of interest}

The authors declare no financial or personal relationship that might inappropriately influence the writing of this paper.

\section{References}

1. Anney VN. Ensuring the quality of the findings of qualitative research: looking at trustworthiness criteria. Journal of Emerging Trends in Educational Research and Policy Studies (JETERAPS) 2014; 5: 272-281.

2. Ary D, Jacobs LC, Sorensen C, Razavieh A. Introduction to Research in Education. $8^{\text {th }}$ ed. Wadsworth Cengage Learning; 2010.

3. Balira R, Mabey D, Weiss H, Ross DA, Changalucha J, WatsonJones D. The need for further integration of services to prevent mother-to-child transmission of HIV and syphilis in Mwanza City, Tanzania. Int J Gynecol Obstet 2015; 130: S51-S57.

4. Bhardwaj S, Carter C, Aarons GA, Chi BH. Implementation research for the prevention of mother-to-child HIV transmission in Sub-Saharan Africa: existing evidence, current gaps, and new opportunities. Curr HIV/AIDS Rep 2015; 12: 246-255.

5. Clouse K, Fox MP, Mongwenyana C, et al. "I will leave the baby with my mother": long-distance travel and follow-up care among HIV-positive pregnant and postpartum women in South Africa. J Int AIDS Soc 2018; 21: e25121.

6. Creswell JW. Research Design: Qualitative, Quantitative, and Mixed Methods Approaches. United States: Sage Publications, Inc.; 2013.

7. Flint A. Traditional healing, biomedicine and the treatment of HIV/AIDS: contrasting South African and Native American experiences. Int J Environ Res Public Health 2015; 12: 4321-4339.

8. Gopalappa C, Stover J, Shaffer N, Mahy M. The costs and benefits of Option $\mathrm{B}+$ for the prevention of mother-to-child transmission of HIV. AIDS 2014; 28: S5-S14.

9. Gourlay A, Birdthistle I, Mburu G, Iorpenda K, Wringe A. Barriers and facilitating factors to the uptake of antiretroviral drugs for prevention of mother-to-child transmission of HIV in sub-Saharan Africa: a systematic review. J Int AIDS Soc 2013; 16: 18588.

10. Iwelunmor J, Ezeanolue EE, Airhihenbuwa CO, Obiefune MC, Ezeanolue CO, Ogedegbe GG. Socio-cultural factors influencing the prevention of mother-to-child transmission of HIV in Nigeria: a synthesis of the literature. BMC Public Health 2014; 14: 771.

11. Katz IT, Tsai AC. Psychological therapy to improve HIV care and reduce stigma. Lancet HIV 2015; 2: e172-e173.

12. Lankowski AJ, Siedner MJ, Bangsberg DR, Tsai AC. Impact of geographic and transportation-related barriers on HIV outcomes in subSaharan Africa: a systematic review. AIDS Behav 2014; 18: 1199-1223.

13. Merga H, Woldermichael K, Dube L. Utilization of prevention of mother-to-child transmission of HIV services and associated factors among antenatal care attending mothers in Sebeta Town, Central Ethiopia. Adv Public Health 2016; 2016: 6250898.

14. Mendoza MD, Lopez M. Culture, race, and ethnicity issues in healthcare. In: Paulman P, Taylor R (eds.). Family Medicine. Cham: Springer; 2015.

15. Morris NK, Du Toit-Prinsloo L, Webber L, Saayman G. The prevalence of HIV in the sudden, unexplained and unexpected death population at the Pretoria Medico-Legal Laboratory. South African Journal of HIV Medicine 2016; 17: a424.

16. McMahon SA, Kennedy CE, Winch PJ, Kombe M, Killewo J, Kilewo C. Stigma, facility constraints, and personal disbelief: why women disengage from HIV care during and after pregnancy in Morogoro Region, Tanzania. AIDS Behav 2017; 21: 317-329.

17. National Department of Health, South African National AIDS Council. Clinical Guidelines: PMTCT (Prevention of Mother-toChild Transmission). Pretoria: NdoH, 2010. Available at: http:// www.fidssa.co.za/Content/Documents/PMTCT_Guidelines.pdf.

18. National Department of Health. National Consolidated Guidelines for the Prevention of Mother-to-child Transmission of HIV (PMTCT) and the Management of HIV in Children, Adolescents and Adults. National Department of Health. Pretoria. April 2015. Available at: https://www.health-e.org.za/2015/07/02/guidelines-nationalconsolidated-guidelines-for-pmtct-and-the-management-of-hiv-inchildren-adolescents-and-adults/.

19. National Department of Health. Annual Performance Plan, 2016/17 - 2018/19. National Department of Health. Pretoria; 2016.

20. Ogbonna K, Govender I, Tumbo J. Knowledge and practice of the prevention of mother-to-child transmission of HIV guidelines amongst doctors and nurses at Odi Hospital, Tshwane District. South African Family Practice 2016; 58: 167-171.

21. Ramashala MR. Factors contributing to Mother To Child Transmission (MTCT) of HIV in Seshego Zone clinics, Capricorn district in South Africa. Unpublished mini-dissertation. Limpopo: University of Venda; 2019.

22. Robinson OC. Sampling in interview-based qualitative research: a theoretical and practical guide. Qual Res Psychol 2014; 11: 25-41.

23. O’Neill K, Takane M, Sheffel A, Abou-Zahr C, Boerma T. Monitoring service delivery for universal health coverage: the Service Availability and Readiness Assessment. Bull World Health Organ 2013; 91: 923-931.

24. Simba D, Kamwela J, Mpembeni R, Msamanga G. The impact of scaling-up prevention of mother-to-child transmission (PMTCT) of HIV infection on the human resource requirement: the need to go beyond numbers. The International Journal of Health Planning and Management 2010; 25: 17-29.

25. Schnake-Mahl AS, Sommers BD. Health care in the suburbs: an analysis of suburban poverty and health care access. Health Affairs 2017; 36: 1777-1785

26. Schensul JJ, Le Compte MD. Essential ethnographic data collection methods: a mixed methods approach. United States of America: Altamira Press; 2013.

27. Spangler SA, Abuogi LL, Akama E, et al. From 'half-dead' to being 'free': resistance to HIV stigma, self-disclosure and support for PMTCT/HIV care among couples living with HIV in Kenya. Cult Health Sex 2018; 20: 489-503.

28. Statistics South Africa. Mid-year population estimates. 2012 (Online). Available at: http://www.statssa.gov.za. (Accessed: 26.08.2015).

29. UNAIDS. Combination HIV prevention: tailoring and coordinating biomedical, behavioural and structural strategies to reduce new HIV infections. Geneva: World Health Organization; 2010.

30. UNAIDS. Global AIDS Update 2016. Geneva: World Health Organization; 2016.

31. UNICEF. Children and AIDS Fifth Stocktaking Report, 2010. Geneva: World Health Organization; 2010.

32. World Health Organization. The global health sector strategy on HIV/AIDS 2011-2015: an interim review of progress: abridged report. Geneva: World Health Organization; 2014.

33. WHO. PMTCT strategic vision 2010-2015: preventing mother-tochild transmission of HIV to reach the UNGASS and Millennium Development Goals. Geneva: World Health Organization; 2010. 